\title{
Foundations of a Unified Physics
}

\section{Pestov IB*}

Bogoliubov Laboratory of Theoretical Physics, Joint Institute for Nuclear Research, 141980, Dubna, Moscow Region, Russia

\begin{abstract}
Since space is the primary concept of science, we put forward an idea that regularities of unified physics are concealed in a simple relation: everything in the concept of space and the concept of space in everything. With this hypothesis as a ground, a conceptual structure of a unified geometrical theory of fundamental interactions is created and deductive derivation of its main equations is produced. The formulated theory provides opportunity to understand the origin and nature of physical fields and local internal symmetry; time and energy; spin and charge; confinement and quark-lepton symmetry; dark energy and dark matter, giving by this solution of the fundamental physical problems and conforming together with this the existence of new physics in its unity.
\end{abstract}

Keywords: Unified physics; Unified geometrical theory; Smooth manifold

\section{Introduction}

The creation of a unified geometrical theory of fundamental interactions is motivated by two severe constraints. It is natural to suppose that solution of the fundamental physical problems cannot be given without a more drastic revision of our fundamental concepts than any that have done before. Quite likely these innovations are so great that direct attempts to derive new ideas from experimental data will be beyond the power of human intelligence [1]. And second, we need to know the origin of the laws and, hence, to formulate a new theory which considers world as a whole (as an integral structure) and gives a possibility to understand why nature is just the way it is. To produce a single theory that provides understanding of the structure of our universe as a unique possible consequence of the only simple assumption (a first principle), we abstract away from the known concepts and laws and put forward the fundamental principle of unification which can be presented in the form of the grand relation: fundamental physical ideas, symmetries and equations are tightly connected with the idea of space and, hence, they have the geometrical origin and are formulated in the form completely independent of any outer and a priori conditions (everything in the concept of space and the concept of space in everything). Thus, the radically new idea of a unified physics is a most possible generality and the outlined first principle. The theory presented is the only one unique logically possible physical theory since it gives the solution of the most difficult and long-standing problem in the history of science - the problem of time. A new concept of time provides the formulation of the law of energy conservation (the most fundamental law of the nature) in the form which is the most general and, hence, suitable for all cases without exclusion. When we discover connections of time with other objects, the reason of the existence of time becomes clear.

The most general realization of the idea of space is the concept of physical manifold. The physical manifold is a smooth manifold [2] plus its connections with physical fields that transfer energy and momentum. The second part of this definition is especially important for understanding of the nature of the gravitational field and time [3,4]. Most physicists nowadays consider a theory be fundamental only if it does make explicit use of the concept of smooth manifold. Thus, the first principle of unification should be formulated as follows: everything in the concept of physical manifold and the concept of physical manifold in everything. With this only first principle we can get the ideas and results we need to describe and understand a realm where all intuitions derived from the life in common space and time become inapplicable.

Some words about mathematics we use. Of course, similar to the electromagnetic field all the geometrical quantities are invariant and, hence, are coordinate free and basis-free. But physical laws are formulated as systems of differential equations in partial derivatives. Hence, the using of the coordinates is desirable. Let us consider a simple example to illustrate our statement. It is clear that number is invariant but we need to remember about the interplay between an observer and computing machines which is provided by the binary number system. We also pay attention on the mathematics of some novel developments of modern physics $[5,6]$.

The super symmetry $[7,8]$, the superstring theory $[5,6]$ and the M-theory [9], were analyzed by Steven Weinberg in his article [10], entitled "A Unified Physics by 2050?" We cite his general conclusion: Observations of this kind will yield valuable clues to the unified theory of all forces, but the discovery of this theory will probably not be possible without radically new ideas." Since the unified physics provides the new understanding of the nature of spin and superstring theories (with the problem of their unification) have no a particular support from the side of experiment, it is natural to reconsider the interesting idea of super symmetry in the framework of the unified physics with its new concept of time and to discover the real underlying degrees of freedom of M-theory.

\section{General Characteristic of Fields and Time}

The geometrical structure of the physical manifold (points, curves, congruence's of curves, families of curves) determines a very restricted set of really geometrical quantities and along with that geometrical internal symmetry that makes these quantities variable and forms from them the fundamental physical fields: the positive definite Riemann metric $g_{i j}$ which is the potential of the gravitational field providing connection of the physical fields with the physical manifold

*Corresponding author: Pestov IB, Bogoliubov Laboratory of Theoretical Physics Joint Institute for Nuclear Research, 141980, Dubna, Moscow Region, Russia, Tel: 74962163695; E-mail: pestov@theor.jinr.ru

Received January 30, 2015; Accepted April 21, 2015; Published April 27, 2015

Citation: Pestov IB (2015) Foundations of a Unified Physics. J Phys Math 6: 135 doi:10.4172/2090-0902.1000135

Copyright: (C) 2015 Pestov IB. This is an open-access article distributed under the terms of the Creative Commons Attribution License, which permits unrestricted use, distribution, and reproduction in any medium, provided the original author and source are credited. 
(it is characterized by the re parameterization invariance); the linear (affine) connection $P_{j k}^{i}$ which is the potential of the generalized electromagnetic field ( the group of geometrical internal symmetry is a general linear group); the scalar and covariant vector fields, and anti-symmetric covariant tensor fields which are connected by the geometrical internal symmetry (spin symmetry) into the real spinning field.

$$
\mathbf{A}=\left(a, a_{i}, a_{i j}, a_{i j k}, a_{i j k l}\right), \quad i, j, k, l=1,2,3,4 .
$$

The intrinsic causal structure of the physical manifold is the temporal field (the natural clock) and bilateral symmetry defined by the stream of time. The temporal field with respect to the coordinate system $u^{1}, u^{2}, u^{3}, u^{4}$ is denoted by $f(u)=f\left(u^{1}, u^{2}, u^{3}, u^{4}\right)$. The reading $t$ of the natural clock is defined by the formula $t=f(u)=f\left(u^{1}, u^{2}, u^{3}, u^{4}\right)$ for any point of the physical manifold. The two events at the point's $u^{1}, u^{2}$, $u^{3}, u^{4}$ and $v^{1}, v^{2}, v^{3}, v^{4}$ go on concurrently if $f\left(u^{1}, u^{2}, u^{3}, u^{4}\right)=f\left(v^{1}, v^{2}, v^{3}\right.$, $\left.v^{4}\right)$. The gradient of the temporal field (the stream of time) is the vector field $t$ with the components $t^{i}=(\nabla f)^{i}=g^{i j} \partial_{j} f=g^{i j} t_{j}$, where $g^{i j}$ are the contravariant components of the Riemann metric $g_{i j}$. The temporal field obeys the fundamental equation:

$$
D_{t} f=(\nabla f)^{2}=g^{i j} \frac{\partial f}{\partial u^{j}} \frac{\partial f}{\partial u^{j}}=1
$$

Which means that time flows equably. This equation has general solution [11] and special solution known as the function of geodesic distance.

The stream of time defines fundamental discrete internal symmetry- bilateral symmetry. A pair of vector fields $v$ and $\mathbf{v}$ has bilateral symmetry if the sum of these fields is collinear to the gradient of a temporal field and their difference is orthogonal to it, $\overline{\mathbf{v}}+\mathbf{v}=\lambda \mathbf{t}$, $(\overline{\mathbf{v}}, \mathbf{t})=(\mathbf{v}, \mathbf{t})$, where $(\mathbf{v}, \mathbf{w})=g_{i j} v^{i} w^{j}=v^{i} w_{i}$ is a scalar product. The bilateral symmetry may be represented as a linear transformation (reflection) $\bar{v}^{i}=R_{j}^{i} v^{j}$, where $R_{j}^{i}=2 t^{i} t_{j}-\delta_{j}^{i}$.

The bilateral symmetry defines the causal structure of the physical manifold and the auxiliary metric

$$
\bar{g}_{i j}=2 t_{i} t_{j}-g_{i j}=g_{i k} R_{j}^{k}, \quad \bar{g}^{i j}=2 t^{i} t^{j}-g^{i j}
$$

Giving the straightforward method of introduction of a temporal field (and together with this the dynamics) into the Lagrangians (and the equations) of the fundamental physical fields $[3,4,11]$.

From the consideration of the bilateral symmetry it follows that in the geometrical (coordinate independent) form the time reversal invariance means that a theory is invariant with respect to the transformations $T: t^{i} \rightarrow-t^{i}$.

It is clear that a theory will be time reversal invariant if the gradient of the temporal field appears in all formulae only as an even number of times, like $t^{i} t^{j}$.

\section{Equations of Gravidynamics}

The difference between general covariance and reparametrization invariance is established and a new mathematical operation of constructing of the new fields by the reparametrization is introduced. The dynamical equations of the gravitational field and the law of energy conservation are represented. The comparison of these equations with the Hamilton equation (which is the cornerstone of the solution of the Poincare conjecture given by Perelman) is discussed.

The manifold $M$ is called topologically nontrivial if it cannot to be maintained by one nonsingular coordinate system. If we insist on one coordinate system, we get into singularities. If we relax that, we have no singularity. In general, the physical manifold is topologically nontrivial. To establish exact mathematical and physical meaning of general covariance and reparametrization invariance, we suppose, for simplicity, that two systems of coordinates are sufficient. Thus, there are two open neighborhoods $U$ and $\bar{U}$ such that $M=U \cup \bar{U}$ and two homeomorphisms of $U$ and $\bar{U}$ onto an open sets of $R^{n}$. In the domain of overlapping $U$ and $\bar{U}, W=U \cap \bar{U}$ two systems of coordinates $u^{1}, u^{2}$, $u^{3}, u^{4}$ and $\bar{u}^{1}, \bar{u}^{2}, \cdots, \bar{u}^{n}$ compete with each other and this takes the form of the functional relations

$$
\bar{u}^{i}=\bar{u}^{i}\left(u^{1}, u^{2}, \cdots, u^{n}\right), \quad u^{i}=u^{i}\left(\bar{u}^{1}, \bar{u}^{2}, \cdots, \bar{u}^{n}\right), \quad i=1,2, \cdots n,
$$

Called coordinate transformations. Let the sets of functions

$$
g_{i j}\left(u^{1}, u^{2}, \cdots, u^{n}\right), \quad \bar{g}_{i j}\left(\bar{u}^{1}, \bar{u}^{2}, \cdots, \bar{u}^{n}\right)
$$

Represent correspondingly the gravitational field in the neighborhoods $U$ and $\bar{U}$. Then, in the simplest case the following relations should be valid in the domain of overlapping $W$

$$
\bar{g}_{i j}=\frac{\partial u^{-k}}{\partial u^{i}} \frac{\partial u^{-l}}{\partial u^{j}} g k l
$$

Thus, under the consideration of the physical fields on the topologically nontrivial manifolds the principle of general covariance holds valid.

Ingeneral, the connection between $g_{i j}\left(u^{1}, u^{2}, \cdots, u^{n}\right), \quad \bar{g}_{i j}\left(\bar{u}^{1}, \bar{u}^{2}, \cdots, \bar{u}^{n}\right)$ can be more refined. To show this, let us consider diffeomorphisms $\varphi$ of $U$ onto itself, which is defined as follows

$$
\begin{aligned}
& \varphi: u^{i} \Rightarrow \varphi^{i}\left(u^{1}, u^{2}, \cdots, u^{n}\right), \quad \varphi^{-1}: u^{i} \Rightarrow \theta^{i}\left(u^{1}, u^{2}, \cdots, u^{n}\right), \\
& \varphi^{i}\left(\theta^{1}(u), \theta^{2}(u), \cdots, \theta^{n}(u)\right)=u^{i}, \quad \theta^{i}\left(\varphi^{1}(u), \varphi^{2}(u), \cdots, \varphi^{n}(u)\right)=u^{i} .
\end{aligned}
$$

The diffeomorphism $\varphi$ induces the mapping $\tilde{g}=\varphi g$ of the form

$$
\tilde{g}_{i j}(u)=g_{k l}(\theta(u)) \theta_{i}^{k}(u) \theta_{j}^{l}(u),
$$

Where $\theta_{j}^{l}(u)=\partial_{j} \theta^{l}(u)$. The last formula describes the process of getting of the new fields by the reparametrization. Hence, in the most general case we instead of equation (2) have the following equation of the analitic continuation

$$
\bar{g}_{i j}=\frac{\partial \bar{u}^{k}}{\partial u^{i}} \frac{\partial \bar{u}^{l}}{\partial u^{j}} \tilde{g}_{k l} .
$$

If $g_{i j}(u)$ and $\tilde{g}_{i j}(u)$ are solutions of some equation, this equation is called invariant with respect to the reparametrization defined by the diffeomorphisms $\varphi$. Inversely, if equation is invariant with respect to reparametrization, it is possible to construct a set of new solutions of the same equation having a given solution. Thus, in the general case to establish connection between $g$ and $\bar{g}$, the intermediate factor $g$ should be taken into account. Reparametrization invariance expresses in the exact mathematical form that in general we do not insist on one gravitational potential $g_{i j}(u)$ in the given coordinate patch. It is very important for the understanding the nature of possible singularities (true or not).

From our consideration it follows that equations of the physical fields on the topologically nontrivial manifolds should be general covariant and invariant with respect to the reparametrization. The simultaneous consideration of these two things is very important since, for example, the Maxwell equations alone are generally covariant but they are not invariant with respect to the reparametrization and this is not the case if we consider the genuine Riemann metric alone. By this the central role of the gravitational field in a unified physics is defined. 
We denote

$$
\Gamma_{j k}^{i}=\frac{1}{2} g^{i l}\left(\partial_{j} g_{k l}+\partial_{k} g_{j l}-\partial_{l} g_{j k}\right), \quad R_{i j k}{ }^{l}=\partial_{i} \Gamma_{j k}^{l}-\partial_{j} \Gamma_{i k}^{l}+\Gamma_{i m}^{l} \Gamma_{j k}^{m}-\Gamma_{j m}^{l} \Gamma_{i k}^{m}
$$

And get the Ricci tensor $R_{j k}=R_{l j k}{ }^{i}$ and covariant derivative $\nabla_{i}$ with respect to $\Gamma_{j k}^{i}$. With this we introduce momentum of the gravitational field

$$
P_{j}^{i}=\frac{1}{2} g^{i k} D_{\mathrm{t}} g_{j k}=\frac{1}{2} g^{i k}\left(\nabla_{j} t_{k}+\nabla_{k} t_{j}\right)=g^{i k} \nabla_{j} t_{k}=\nabla_{j} t^{i},
$$

Where $D_{t}$ is the Lie derivative along a stream of time. The stress tensor of the gravitational field can be written in the following form:

$$
S_{j}^{i}=h_{k}^{i} R_{l}^{k} h_{j}^{l}+D_{\mathbf{t}} P_{j}^{i}+\varphi P_{j}^{i},
$$

Where $\varphi=\nabla_{i} t^{i}$ and $h_{j}^{i}=\delta_{j}^{i}-t_{j} t^{i}$. for a density of kinetic and potential energy of the gravitational field we have the following expressions:

$$
\begin{aligned}
& T=\frac{1}{2}\left(P_{i}^{i}\right)^{2}-\frac{1}{2} P_{j}^{i} P_{i}^{j}=\frac{1}{2}(\operatorname{Tr} P)^{2}-\frac{1}{2} \operatorname{Tr}\left(P^{2}\right), \\
& U=\frac{1}{2} S=\frac{1}{2} S_{l}^{l} .
\end{aligned}
$$

The first group of equations of Gravidynamics reads

$$
D_{t} P_{j}^{i}+\varphi P^{i}{ }_{j}+S^{i}{ }_{j}+N^{i}{ }_{j}=\frac{1}{2} \varepsilon h^{i}{ }_{j} \text {, }
$$

Where $N_{i}^{i}$ is the stress tensor of the generalized electromagnetic field and spinning field, ${ }^{\mathcal{E}}$ is the energy density of the gravitational field and other fields $\varepsilon=\varepsilon_{g}+\varepsilon_{m}$ The second group of equations of Gravidynamics reads

$\mathrm{G}_{j}=\Pi_{i}$,

Where

$$
\mathrm{G}_{j}=h_{j}^{i}\left(\nabla_{l} P_{i}^{l}-\partial_{i} P_{l}^{l}\right)
$$

is the energy flow vector of the gravitational field and $\Pi_{i}$ is the energy flow vector of the other fields. We pay attention to that the heat equation of Hamilton (tightly connected with the solution of the Poincare conjecture) can be written in our notation as follows:

$$
P_{j}^{i}+S_{j}^{i}=0
$$

Equation (3) can be derived from the energy functional $L=T-U$ and according to the Hamilton paper; this equation is more natural than his heat equation. Thus, we have there very interesting physical and mathematical problem to consider connection between gravity and topology in the new framework.

The law of energy conservation reads.

$$
D_{\mathrm{t}}(\sqrt{g} \varepsilon)=0, \quad \varepsilon=\varepsilon_{\mathrm{g}}+\varepsilon_{\mathrm{m}}=T+U+\varepsilon_{\mathrm{m}} .
$$

Since the scalar temporal field enters into the Lagrangians of the physical fields in the form of the gradient of the scalar field

$$
t_{i}=\partial_{i} f(u),
$$

The laws of a unified physics are invariant with respect to transformations of the form

$$
f(u) \Rightarrow f(u)+a
$$

Where $a$ is a constant, this is internal symmetry that defines the law of energy conservation as a fundamental physical law of the universe which is true in all cases.

\section{Equations of Generalized Electromagnetic Field}

Here we establish dynamical equations of the generalized electromagnetic field (shortly gef) which are defined by gef symmetry and describe a new form of matter which interacts only gravitationally (dark matter) [12-14]. The problem of invisible mass is acknowledged to be among the greatest puzzles of modern cosmology and field theory. The most direct evidence for the existence of large quantities of dark matter in the Universe comes from the astronomical observation of the motion of visible matter in galaxies. One neither knows the identity of dark matter nor whether there are one or more types of its structure elements. The most commonly discussed theoretical elementary particle candidates are a massive neutrino, a syper symmetric neutralino, and the axion. So, at the present time there is a good probability that the set of known fields is by no means limited to those fields. Moreover, we are free to look for deeper reasons for the existence of a new form of energy unusual in many respects.

The parallel displacement of vector fields $\overline{\mathbf{v}}$ and $V$ with bilateral symmetry $\bar{v}^{i}=R_{j}^{i} v^{j}$ can be produced only by a pair of connections $\bar{P}_{j k}^{i}$ and $P_{j k}^{i}$ with bilateral symmetry. From the law of parallel displacement we have

$$
\bar{P}_{j k}^{i}=R_{m}^{i} P_{j n}^{m} R_{k}^{n}+R_{m}^{i} \partial_{j} R_{k}^{m} .
$$

Being generalized the bilateral symmetry takes status of gef symmetry $G L(n, R)$ with the law of transformation

$$
\bar{P}_{j k}^{i}=U_{m}^{i} P_{j n}^{m} V_{k}^{n}+U_{m}^{i} \partial_{j} V_{k}^{m},
$$

Where $V^{i}$ is the component of the operator $U^{-1}$ inverse to the operator $U, U_{k}^{i} V_{j}^{k}=\delta_{j}^{i}$. For brevity, we use the matrix notation

$$
\mathbf{U}=\left(U_{l}^{k}\right), \quad \mathbf{P}_{i}=\left(P_{i l}^{k}\right), \quad \mathbf{E}=\left(\delta_{l}^{k}\right), \quad \mathbf{H}_{i j}=\left(H_{i j l}{ }^{k}\right), \quad \operatorname{Tr} \mathbf{U}=U_{k}^{k} .
$$

The transformations of gef symmetry take the form

$$
\overline{\mathbf{P}}_{i}=\mathbf{U} \mathbf{P}_{i} \mathbf{U}^{-1}+\mathbf{U} \partial_{i} \mathbf{U}^{-1}=\mathbf{P}_{i}+\mathbf{U D}_{i} \mathbf{U}^{-1},
$$

Where $\mathrm{D}_{i}$ is the natural differential operator associated with gef symmetry only

$$
\mathrm{D}_{i} \mathbf{U}=\partial_{i} \mathbf{U}+\mathbf{P}_{i} \mathbf{U}-\mathbf{U} \mathbf{P}_{i}=\partial_{i} \mathbf{U}+\left[\mathbf{P}_{i}, \mathbf{U}\right] .
$$

The Riemann tensor of $P_{j k}^{i}$

$$
\mathbf{B}_{i j}=\partial_{i} \mathbf{P}_{j}-\partial_{j} \mathbf{P}_{i}+\left[\mathbf{P}_{i}, \mathbf{P}_{j}\right]
$$

is reducible with respect to the transformations

$$
\overline{\mathbf{B}}_{i j}=\mathbf{U} \mathbf{B}_{i j} \mathbf{U}^{-1} \text {, }
$$

Since

$$
\mathbf{B}_{i j}=\left(\mathbf{B}_{i j}-\frac{1}{4} \operatorname{Tr}\left(\mathbf{B}_{i j}\right) \mathbf{E}\right)+\frac{1}{4} \operatorname{Tr}\left(\mathbf{B}_{i j}\right) \mathbf{E} .
$$

Hence, the strength tensor of the generalized electromagnetic field is given by the formula

$$
\mathbf{H}_{i j}=\mathbf{B}_{i j}-\frac{1}{4} \operatorname{Tr}\left(\mathbf{B}_{i j}\right) \mathbf{E}, \quad \operatorname{Tr}\left(\mathbf{H}_{i j}\right)=0
$$

and the singlet state of the gef defines the strength tensor of the electromagnetic field $F_{i j}=\operatorname{Tr}\left(\mathbf{B}_{i j}\right)$.

The ground state of gef is defined by the equation $B_{i j}=0$ which means that this state transfers a new form of energy. We give general solution of this equation. Let four linear independent vector fields $E^{\mu}$ be given and one can construct purely algebraically components of the four covector fields $E^{\mu}{ }_{i}$, so that $E_{\mu}^{i} E_{j}^{\mu}=\delta_{j}^{i}$ holds valid. Setting $P_{j k}^{i}=L_{j k}^{i}$, where $L_{j k}^{i}=E_{\mu}^{i} \partial_{j} E_{k}^{\mu}$, get general solution of the equation 
in question. Let us introduce a tensor field $Q_{j k}^{i}=P_{j k}^{i}-L_{j k}^{i}$ but in what follows we shall consider the irreducible deviation tensor

$$
T_{j k}^{i}=Q_{j k}^{i}-\frac{1}{4} Q_{j l}^{l} \delta_{k}^{i}, \quad \mathbf{T}_{j}=\mathbf{Q}_{j}-\frac{1}{4} \operatorname{Tr}\left(\mathbf{Q}_{j}\right) \mathbf{E} .
$$

Let us introduce electric and magnetic strength of the generalized electromagnetic field by the direct and inverse mapping

$$
\begin{aligned}
& \mathbf{J}_{i}=t^{k} \mathbf{H}_{i k}, \quad \mathbf{M}_{i}=\frac{1}{2} e_{i k j l} t^{k} \mathbf{H}^{j l}=t^{k} \stackrel{*}{\mathbf{H}} \\
& \mathbf{H}_{i k}=-t_{i} \mathbf{J}_{k}+t_{k} \mathbf{J}_{i}-e_{i k j l} t^{j} \mathbf{M}^{l},
\end{aligned}
$$

The Lagrangian of gefdynamics takes the form

$$
\mathcal{L}_{p}=-\frac{1}{4} \operatorname{Tr}\left(\mathrm{H}_{i j} \tilde{\mathrm{H}}^{i j}\right)-\frac{\mu^{2}}{2} \operatorname{Tr}\left(\mathrm{T}_{i} \tilde{\mathrm{T}}^{i}\right)
$$

Where $\mu$ is a constant of dimension of $\mathrm{cm}^{-1}$

$$
\tilde{\mathbf{H}}^{i j}=\bar{g}^{i k} \bar{g}^{j l} \mathbf{H}_{k l}=2 t^{i} \mathbf{J}^{j}-2 t^{j} \mathbf{J}^{i}+\mathbf{H}^{i j}, \tilde{\mathbf{T}}^{i}=\bar{g}^{i k} \mathbf{T}_{k} .
$$

The first pair of equations of gefdynamics can be represented in the following form:

$$
\begin{aligned}
& t^{k} \mathrm{D}_{k} \mathbf{M}^{i}-\mathbf{M}^{k} \partial_{k} t^{i}+\varphi \mathbf{M}^{i}=-e^{i j k l} t_{j} \mathrm{D}_{k} \mathbf{J}_{l}, \\
& \frac{1}{\sqrt{g}} \mathrm{D}_{i}\left(\sqrt{g} \mathbf{M}^{i}\right)=0,
\end{aligned}
$$

where $\varphi=\nabla_{i} t^{i}$.

The second pair of equations of gefdynamics reads

$$
\begin{aligned}
& t^{k} D_{k} \mathbf{J}^{i}-\mathbf{J}^{k} \partial_{k} t^{i}+\varphi \mathbf{J}^{i}=e^{i j k l} t_{j} \mathrm{D}_{k} \mathbf{M}_{l}+\mu^{2} \mathbf{S}^{i}, \\
& \frac{1}{\sqrt{g}} \mathrm{D}_{i}\left(\sqrt{g} \mathbf{J}^{i}\right)=\mu^{2} \mathbf{S},
\end{aligned}
$$

Where

$$
\mathbf{S}_{i}=h_{i}^{j} \mathbf{T}_{j}, \quad \mathbf{S}=t^{k} \mathbf{T}_{k}
$$

Since

$$
\operatorname{Tr}\left(\mathbf{J}_{i}\right)=\operatorname{Tr}\left(\mathbf{M}_{i}\right)=\operatorname{Tr}\left(\mathbf{T}_{i}\right)=0,
$$

The system of equations in question is simultaneous. This system of equations describes a new form of matter which is the structure element of the unified physics and has the characteristics of an entity known from the observations as Dark Matter.

\section{Equations of Spin Dynamics}

Spin is already a great unifying principle in the theoretical physics, but its potential is far from being exhausted. In a Unified Physics the Spin dynamics includes the region of physical phenomena which are now investigated in the Standard Model. Thus, spin symmetry is geometrical internal symmetry which provides new and complete understanding of spin and, hence, theory of elementary particles as well. Here we derive equations of Spin dynamics and with this process introduce operators of electrical charge and neutrino charge and discuss some questions connected with this innovation [16].

The spin symmetry group is a general linear group $G L(16, R)$ that acts in the space of the spinning fields. We constructed a natural general covariant basis in the Lie algebra $g l\left(2^{n}, R\right)$ of $G L\left(2^{n}, R\right)$ and uncover spin as the bipolar structure on the group of spin symmetry $G L$ $\left(2^{n}, R\right)[11]$. The bipolar structure having two dual sets of commuting operators exists which define the Lie algebra of two dual groups $S$ and $\tilde{S}$. This result expresses in the mathematical form that the spinning field has internal angular momentum and magnetic momentum and spinning field is the space of the two-valued representation of the dual groups $S$ and $\tilde{S}$.

Let us define the positive definite scalar product and the auxiliary scalar product in the linear space in question as follows:

$$
\begin{aligned}
& \langle\mathbf{A} \mid \mathbf{B}\rangle=\sum_{p=0}^{4} \frac{1}{p !} a_{i_{1} \cdots i_{p}} b_{j_{1} \cdots j_{p}} g^{i_{1} j_{1}} \cdots g^{i_{p} j_{p}}, \\
& \langle\mathbf{A} \mid \mathbf{B}\rangle=\sum_{p=0}^{4} \frac{1}{p !} a_{i_{1} \cdots i_{p}} b_{j_{1} \cdots j_{p}} \bar{g}^{i_{1} j_{1}} \cdots \bar{g}^{i_{p} j_{p}} .
\end{aligned}
$$
valid:

The following relation between these two scalar products holds

$$
\langle\mathbf{A} \mid \mathbf{B}\rangle=(\mathbf{A} \mid \mathbf{R B}),
$$

Where

$$
\mathrm{R}=-Q_{\mathrm{t}} \tilde{Q}_{\mathrm{t}}=Q_{\mathrm{t}} \tilde{Q}_{\mathrm{t}}=Q_{\mathrm{t}} \tilde{Q}_{\mathrm{t}}
$$

And

$$
\begin{aligned}
& Q_{\mathbf{t}}: \bar{a}_{i_{1} \cdots \cdots i_{p}}=p t_{\left[i_{1}\right.} a_{\left.i_{2} \cdots i_{p}\right]}-t^{k} a_{k_{1} \cdots i_{p}}, \\
& Q: \bar{a}_{i_{1} \cdots i_{p}}=(-1)^{p}\left(p t_{\left[i_{1}\right.} a_{\left.i_{2} \cdots i_{p}\right]}+t^{k} a_{k i_{1} \cdots i_{p}}\right), \quad(p=0,1,2,3,4),
\end{aligned}
$$

The square brackets [...] denote the process of alternation and $t^{i}=g^{i j} t_{j}$ The operator R gives the straight forward representation of the bilateral symmetry and shows that this representation opens a new possibility to treatise the bilateral symmetry in the case of the spinning field. We can also define that a system of two spinning fields $A$ and $\overrightarrow{\mathbf{A}}$ possesses the bilateral symmetry if

$$
\overline{\mathbf{A}}=Q_{\mathbf{t}} \mathbf{A}, \text { or } \quad \overline{\mathbf{A}}=Q_{\mathbf{t}} \mathbf{A} \text {. }
$$

In view of this, let us consider two skew-symmetrical bilinear forms

$[\mathbf{A}, \mathbf{B}]=\left(Q_{\mathbf{t}} \mathbf{A} \mid \mathbf{B}\right), \quad[\tilde{\mathrm{A}}, \tilde{\mathrm{B}}]=\left(\tilde{Q}_{\mathbf{t}} \mathbf{A} \mid \mathbf{B}\right)$.

Since $[\mathbf{A}, \mathbf{A}]=0, \quad[\tilde{\mathbf{A}}, \tilde{\mathbf{A}}]=0$,

It is clear that the introduction of this causal structure is adjoined with the consideration of two independent real spinning fields. To understand this new situation it is important to recognize the following results.

Symplectic scalar products are invariant with respect to the transformations of the group $S L(2, R)$ called in what follows the group of pseudo charge symmetry. The generators of this group $h_{1}, h_{2}, h_{3}$ are represented by the real matrices $\tau_{1}, \tau_{2}, \tau_{3}$ which satisfy the following relations:

$$
\tau_{1}^{2}=-1, \quad \tau_{2}^{2}=1, \quad \tau_{1} \tau_{2}+\tau_{2} \tau_{1}=0, \quad \tau_{1} \tau_{2}=\tau_{3}, \quad h_{a}=\frac{1}{2} \tau_{a}, \quad a=1,2,3 .
$$

We consider the matrix $\tau_{1}=Q_{p}$ as an operator of pseudo charge, since its eigenvalues are equal to $\pm i$. In what follows we should like to deal with eigenvectors of this operator and, hence, with a complex spinning field and its conjugated.

$$
\Psi=\frac{\sqrt{2}}{2}(\mathbf{A}+i \mathbf{B}), \quad \stackrel{*}{\Psi}=\frac{\sqrt{2}}{2}(\mathbf{A}-i \mathbf{B})
$$

(as a manifestation of broken pseudo charge symmetry). It is very important to recognize that this provides the existence of the probability 
measure in the space of solutions of the spin dynamics equations for the complex spinning field [11].

The Lagrangian of Spin dynamics associated with this new representation of bilateral symmetry

$$
\mathcal{L}_{\mathrm{t}}=-\frac{i}{2}\left\langle\tilde{Q}_{t} \stackrel{*}{\Psi} \mid \Pi \Psi\right\rangle+\frac{i}{2}\langle\tilde{Q} \Psi \mid \Pi \stackrel{*}{\Psi}\rangle+i m\left\langle\tilde{Q}_{t} \stackrel{*}{\Psi} \mid \Psi\right\rangle,
$$

is not invariant with respect to time reversal $T: t_{i} \rightarrow-t_{i}$, since $L_{\mathrm{t}}=-L_{\mathrm{t}}$. For simplicity we do not write out the dual Lagrangian [11]. The main differential operators of Spin dynamics $\Pi$ and $\tilde{\Pi}$ are defined here by the new geometrical object, fundamental connection of Spin dynamics LOO

$$
\bar{\Gamma}_{j k}^{i}=\Gamma_{j k}^{i}+t^{i} \nabla_{j} t_{k}-t_{k} \nabla_{j} t^{i}
$$

This is characterized by the equations

$$
\bar{\nabla}_{i} t_{j}=0, \quad \bar{\nabla}_{i} g_{j k}=0, \quad \bar{\nabla}_{i} g^{j k}=0,
$$

where $\bar{\nabla}_{i}$ is the covariant derivative with respect to this connection. The operator $\Pi$ is defined as follows:

$$
\text { (ПА) })_{i_{1} \cdots i_{p}}=p \bar{\nabla}_{\left[i_{1}\right.} a_{\left.i_{2} \cdots i_{p}\right]}+\bar{\nabla}^{k} a_{k i_{1} \cdots i_{p}}-2 t^{k} \bar{\nabla}_{\mathbf{t}} a_{k i_{1} \cdots i_{p}},
$$

since $\bar{g}^{k l} \bar{\nabla}_{\mathcal{M}}=2 t^{k} \bar{\nabla}_{\mathrm{t}}-\bar{\nabla}^{k}$. For simplicity, we do not consider the dual operator $\prod_{\text {. }}$.

Equation $\delta L_{\mathbf{t}}=0$ can be written as

$$
\Pi \Psi+\frac{1}{2} \varphi Q_{t} \Psi=m \Psi,
$$

and complex conjugated, where $\varphi=\nabla_{i} t^{i}$.

The Lagrangian of Spin dynamics associated with the customary representation of bilateral symmetry and invariant with respect to the time reversal takes the form

$$
\mathcal{L}_{t}=\frac{1}{2}\langle\mathbf{A} \mid \Pi \mathbf{A}\rangle-\frac{m}{2}\langle\mathbf{A} \mid \mathbf{A}\rangle
$$

Equation $\delta L_{\mathrm{t}}=0$ can be written as follows:

$$
\prod A+\frac{1}{2} \varphi Q_{t} A=m A,
$$

Since

$$
\Pi \tilde{Q}_{\mathbf{t}}-\tilde{Q}_{\mathbf{t}} \Pi=0, \quad\left\langle\tilde{Q}_{\mathbf{t}} \mathbf{A} \mid \mathbf{B}\right\rangle=-\left\langle\mathbf{A} \tilde{Q}_{\mathbf{t}} \mathbf{B}\right\rangle,
$$

the Lagrangian (10) is invariant with respect to the transformations

$$
\overline{\mathbf{A}}=\exp \left(\alpha \tilde{Q}_{\mathbf{t}}\right) \mathbf{A} .
$$

For the density of the corresponding conserved current $C^{k}$ one can find the expression

$$
\rho=t_{i} C^{i}=\left\langle Q_{\mathbf{t}} \mathbf{A} \mid \tilde{Q}_{\mathbf{t}} \mathbf{A}\right\rangle=(\mathbf{A} \mid \mathbf{A})
$$

This shows that there is a natural probability measure in the space of solution of equation (11). This fundamental result was originally recognized in [17] and now it gives us an opportunity to identify the operator $\tilde{Q}_{\mathrm{t}}$ with an operator of the electrical charge $\tilde{Q}_{\mathrm{t}}=Q_{e}$.

Let $A$ be components of the potential of the electromagnetic field a. The Lagrangian of interaction of the real spinning field with the electromagnetic field is

$$
\mathcal{L}_{\text {int }}=-\frac{1}{2} q A_{i} c^{i}=-\frac{1}{2} q \phi \rho-\frac{1}{2} q \phi_{i} J^{i}=-\frac{1}{2} q\left\langle L_{a} A \mid \tilde{\mathrm{Q}}_{t} \mathrm{~A}\right\rangle
$$

Where $q$ is the constant of interaction and

$$
\phi=t^{i} A_{i}, \quad \Phi_{i}=A_{i}-\phi t_{i}, \quad t^{i} \Phi_{i}=0
$$

is, respectively, scalar and vector potential of the electromagnetic field. Equation

$$
\left(\Pi+\frac{1}{2} \varphi Q_{t}\right) A=m A, \mathrm{qL}_{a} \tilde{Q}_{\mathrm{t}} A=m A
$$

Describes electromagnetic interactions and can be considered as a basis for chemistry because the real spinning field carries only electrical charge. For comparison, the Lagrangian (7) is a basis for the strong interaction [11]. The operator $L_{a}$ is define as follows: $L_{\mathbf{a}}=Q_{\mathbf{a}} Z-(\mathbf{t}, \mathbf{a})\left(Q_{\mathbf{t}} Z-Q_{\mathbf{t}}\right)$, where the numerical diagonal operator is introduced $Z: \bar{a}_{i_{1} \cdots i_{p}}=(-1)^{p} a_{i_{1} \cdots i_{p}}, \quad p=0,1,2,3,4$.

The operator of the neutrino charge $Q$ should be connected with the parity non-conservation and, hence, it is defined by the orientation of the physical manifold. The orientation is the anti-symmetrical tensor $e_{i j k l}$ ("element of volume") normalized as $e_{1234}=\sqrt{ } g$, where $g=\operatorname{Det}\left(g_{i j}\right)$.

We define the operator $\tilde{H}$ in the component form

$$
(\tilde{H} \mathbf{A})_{i_{1} \cdots i_{p}}=\frac{1}{(4-p) !}(-1)^{\frac{p(p+1)}{2}} e_{i_{i} \cdots i_{p} j_{1} \ldots j_{4-p}} a^{j_{1} \ldots j_{4-p}}
$$

and derive the following relations:

$$
\tilde{H}^{2}=E, \quad \tilde{H} \tilde{Q}_{\mathbf{t}}+\tilde{Q}_{\mathbf{t}} \tilde{H}=0, \quad \tilde{H} Q_{\mathbf{t}}=Q_{\mathbf{t}} \tilde{H}=0 .
$$

The operator of the neutrino charge $Q_{\nu}$ takes the form

$Q_{v}=\tilde{Q}_{\mathrm{t}} \tilde{H} Q_{\mathrm{t}}$.

The operators $Q_{e}$ and $Q_{v}$ anticommute

$$
Q_{e} Q_{v}+Q_{v} Q_{e}=0, \quad Q_{e}^{2}=Q_{v}^{2}=-E .
$$

The operators $Q e, Q v$ and $Q e v=Q_{e} Q_{v}$ define the representation of $S U$ (2) group. The Lagrangian of Spin

Dynamics (8) is invariant with respect to the transformations

$$
\bar{\Psi}=\exp \left(\alpha Q_{e}\right) \Psi, \quad \bar{\Psi}=\exp \left(\beta Q_{v}\right) \Psi, \quad \bar{\Psi}=\exp \left(\gamma Q_{e v}\right) \Psi,
$$

But it is remarkable that the Lagrangian (10) is invariant only with respect to the transformation generated by the operator of the electric charge. We see that in one case the spinning field carries only the electrical charge but in the second case it carries the electrical charge, the neutrino charge and pseudo charge. In both the cases the spinning field carries the internal spin which is generated by the geometrical internal symmetry defined by the anti -symmetrical tensor field $S_{i j}$ obeying the equation $\bar{\nabla}_{i} S_{j k}=0$. We have no possibility to discuss here this important symmetry [11] to be anywhere in detail.

\section{Conclusions}

Let us mention some results of the Unification. Of course, the principal result is the dynamical equations of the gravitational field, spinning field and generalized electro-magnetic fields which form the nucleus of the unified physics as a system and this system will reveal its internal content in the process of its exploitation. In addition, we should like to formulate the following theses as the evidence that New Physics is the Unified Physics.

1. It is discovered that in the Universe there are two causal structures (the two natural clocks). One of them is defined by general solution of the equation of temporal field (1). Another solution and a new causal structure emerge as special solution of this equation. The new causal 
structure gives natural explanation of the confinement and the baryon number conservation. Indeed, we cannot invoke the artificial concept of force to explain the confinement because for any force there is a more powerful one. But causality is something else again. The baryon number conservation simply expresses in the symbolic form the existence of the second causal structure and nothing else. The quark lepton symmetry means that leptons live in the area of the usual causal structure and quarks live in the area of the new causal structure. Mutual transitions are possible.

2. The role of the gravitational field in the physics of elementary particles is recognized since the polarization of spin symmetry (spin) cannot be discovered without the gravitational field. Spin dynamics can be considered as a new purely rational realization of the Standard Model derived from the only first principle. The observed generations of quarks and leptons are different states of the complex spinning field and the number of these generations equals four. The spinning field describes the physical entity retaining the elementarity and is considered to be characterized solely by equation (12), electrical charge and the internal spin in one case and equation (9), pseudo charge, electric charge, neutrino charge and the internal spin in the other case.

3. The problem of Dark Energy is solved. Dark Energy is simply the energy of the gravitational field.

4. The problem of Dark Matter is solved. The Dark Matter is heavy light that inter-acts only gravitationally. Heavy photons are quants of the generalized electromagnetic field. Massless photons represent a singlet state of this field.

5. The most general and universal law of energy conservation is discovered which holds valid in all cases and is an opportunity for a new contributor of energy.

\section{References}

1. Dirac PAM (1931) Quantised Singularities in the Electromagnetic Field. Proc Roy Soc 60

2. Bernard F, Schutz (1982) Geometrical methods of mathematical physics. Cambridge University Press.

3. Pestov IB (2005) In: Horizons in World Physics, Vol. 248 by A. Reimer, Nova Science, New York.

4. Pestov IB (1996) New concept of time and gravity.

5. Green MB, Schwarz JH, Witten E (1987) Superstring Theory volume 1, Cambridge University Press.

6. Green MB, Schwarz JH, Witten E (1987) Superstring Theory volume 2, Cambridge University Press.

7. Weinberg S (2000) The Quantum Theory of Fields. Cambridge University Press.

8. Smolin L (2006) The Trouble with Physics. Houghton Mifflin.

9. Miemiec A, Schnakenburg I (2006) Basics of M-Theory. Fortsch Phys 54: 5-72.

10. Weinberg $S$ (1999) Scientific American.

11. Pestov IB (2011) Physics of Atomic Nuclei 74: 1084

12. Pestov IB (2000) Gauge theory of oriented media. J Phys A: Math Gen 33 3027.

13. Pestov IB (2006) In: Dark Matter: New Research. Ed. by Val Blain Nova Science, New York.

14. Pestov IB (2013) Geometrization of the Electromagnetic Field and Dark Matter Physics of Particles and Nuclei 44: 442-449.

15. De Rham G (1955) Varietes Differentiable.

16. Hamilton RS (1982) Three-manifolds with positive Ricci curvature. Journ of Diff Geometry 17: 255-306.

17. Pestov IB (1978) Relativistic equations determined by the operators of the exterior derivative and generalized divergence. Theor and Math Phys 34: 48-58.

Submit your next manuscript and get advantages of OMICS Group submissions

Unique features:

User friendly/feasible website-translation of your paper to 50 world's leading languages

Audio Version of published paper

Digital articles to share and explore

Special features:

400 Open Access Journals

30,000 editorial tean

21 days rapid review process

Quality and quick editorial, review and publication processing

Indexing at PubMed (partial), Scopus, EBSCO, Index Copernicus and Google Scholar etc

Sharing Option: Social Networking Enabled

Authors, Reviewers and Editors rewarded with online Scientific Credits

Better discount for your subsequent articles

Submit your manuscript at: http://www.omicsonline.org/submission/ 